\title{
TURNOS TÉCNICO Y ECONÓMICO DE TALA PARA ARBOLES DE ROMERILLO BLANCO EN ECUADOR
}

\section{TECHNICAL AND ECONOMIC TIME POINTS OF LOGGING FOR “ROMERILLO BLANCO” TREES IN ECUADOR}

\author{
Jesús A. Bonilla de Gracia ${ }^{1}$ y Jorge A. Alarcón Novoa ${ }^{2}$
}

\begin{abstract}
Resumen
El presente estudio es el primer trabajo de economía forestal que se ha realizado en los bosques de Numbala, Provincia de Zamora Chinchipe, Ecuador. Por el desconocimiento en torno al inventario forestal del romerillo blanco en la zona, así como el persistente deterioro del bosque en la Reserva Numbala, el presente trabajo de investigación apunta a determinar las edades óptimas de tala, que permitirían un uso sustentable del bosque. Para determinar el óptimo económico se usaron dos modelos: uno basado en el de Fisher-Hotelling $(\mathrm{F}-\mathrm{H})$ y el otro en base a la propuesta de Faustmann-Pressler-Ohlin (F-P-O). Ambos modelos consideraron el nivel de extracción de madera de Schumacher (ecuación bio-forestal funcional que presentó mejor significancia estadística) y una tasa de descuento del 1\%. Para un "stand" de bosque de romerillo blanco, los resultados reportaron una edad óptima de tala económica de 101 años y un valor presente neto de 1164.2 \$ Ha. en el caso del modelo F-H; sin embargo, con el modelo F-P-O, la edad de corte fue de 69 años con un valor presente neto de 1371.1\$/Ha. Se podría concluir que el intervalo de los óptimos económicos resultantes "abarcan" el turno técnico óptimo también estimado, y se ubican en el rango de 69 - 101 años. El turno técnico óptimo, según la función Schumacher, fue estimado en 77 años.
\end{abstract}

Palabras clave: Retrophyllum rospigliosii, valores óptimos de tala, modelo forestal, modelo económico, maximización de beneficios.

\begin{abstract}
This is the first report on forestry economics conducted in the forests of Numbala, Zamora Chinchipe, Ecuador. Due to scarce knowledge on Retrophyllum rospigliosii forest inventory and to the persistent exploitation and deterioration of the Numbala Reserve forest, this research focused in determining the optimal age of logging that would allow sustainable forest use of this plantation. The Fisher-Hotelling (F-H) and the Faustmann-Pressler-Ohlin (F-P-O) models were used to achieve two bio-economic equilibrium results. Both models considered the Schumacher level of logging (bio-forest functional equation which presented best statistical significance), and a discount rate of $1 \%$. Results reported an optimal economic age (in a forest stand) of 101 years, with a net present value of $\$ 1,164.2 \mathrm{per} / \mathrm{Ha}$ in the case of the F-H model; however, the F-P-O economic model reported a cutoff age of 69 years and a net present value of $\$ 1,371.1$ per Ha. It is concluded finally that the range of the resulting economic optimal ages "included" and (also estimated) optimal forest turn, and lie between 69-101 years. The optimal technical age, based on the Schumacher function, was estimated at 77 years.

Key words: Retrophyllum rospigliosii, optimal logging age, economic model, optimal economic logging, profit maximization.
\end{abstract}

\section{Introducción.}

El romerillo blanco (Retrophyllum rospigliosii (Pilg). C. N. Page) es un árbol perteneciente a la familia de las Podocarpaceae; crece en Ecuador, Perú, Colombia, Venezuela, Bolivia y Suecia. En Ecuador se registra a partir de $\operatorname{los} 10^{\circ}$ de latitud sur, en tipos de bosques muy húmedo montano bajo (BMhmb) (Gálvez et al., 2003). De las seis especies registradas del género Retrophyllum, solo una se encuentra en
Ecuador, específicamente en el Cantón Palanda, parroquia Valladolid, Reserva Numbala (Zamora, Chinchipe). La especie es de lento crecimiento y desarrolla en precipitaciones anuales mayores a 1000 $\mathrm{mm}$, altitudes entre 500 a $3600 \mathrm{~m}$., temperaturas entre 8 y $18{ }^{\circ} \mathrm{C}$, humedad relativa 92 a $98 \%$ (Gálvez et al., 2003; Bonilla, 2005).

Estudios realizados en los bosques de Numbala han tenido como resultado que los árboles de 
romerillo blanco crecen muy bien en suelos de textura franco arenoso, $\mathrm{pH}$ alrededor de 3.9, materia orgánica 9.99 y nitrógeno total $118108 \mathrm{ug} / \mathrm{ml}$ (Gálvez et al., 2003). El rendimiento de esta especie varía según las condiciones edáficas, climáticas y disponibilidad de nutrientes, luz solar (poca en los primeros años) y manejo silvicultural (Trujillo, 2007).

El romerillo blanco posee una gran cantidad de usos, como la fabricación de muebles, puertas, pisos, encofrado, ventanas, estructuras aéreas, carpintería, contrachapados y molduras (Lojan, 1992; PREDESUR, 1975). También ha sido considerada de gran potencial como materia prima para la fabricación de papel y recomendada para la recuperación $y$ colonización de suelos erosionados (debido a su capacidad de fijar nitrógeno atmosférico), protección de cuencas hidrográficas, de fauna silvestre, biodiversidad y proveedor de calidad escénica (Rojas, 2005).

Un bosque puede considerarse como proveedor multifuncional, sin embargo este estudio se centra en el aprovechamiento del bosque como proveedor de recurso maderero. Por otro lado, en el análisis de gestión de bosques, un aspecto importante consiste en determinar cuándo se deben talar los árboles desde la óptica forestal y de la economía. Para responder a esta pregunta, desde la óptica forestal, los especialistas emplean modelos que incluyen funciones de crecimiento forestal ${ }^{1}$, considerando variables dasométricas como el DAP (diámetro a la altura del pecho), área basal $(\mathrm{G})$, altura comercial $(\mathrm{HC})$, altura total $(\mathrm{HT})$, factor de forma $(\mathrm{F})$, volumen de madera (V) y edad del árbol (EA). Para el caso de especies maderables de bosques naturales heterogéneos en edades y de regeneración natural, los silvicultores, a partir de un muestreo de edades (método de reconocimiento de anillos), pueden emplear diversas formas funcionales alométricas que permiten pronosticar las edades de toda la población de árboles.

Como la solución del modelo forestal ignora criterios económicos, economistas han considerado el uso de variables económicas para encontrar un turno forestal eficiente; estas variables son precios de madera, costos de suelo, plantación y de cosecha, ingresos, tasas de descuento, entre otras. Los modelos económicos ${ }^{2}$ de optimización usan, entre otros, casos que consideran el turno forestal del valor actual neto (TVAN) y turno del valor esperado de la de la tierra

\footnotetext{
${ }^{1}$ Modelo forestal: contiene únicamente variables biológicoforestales (edad de árboles, altura comercial del árbol, diámetros con y sin corteza de árboles, etc.). Utiliza funciones de crecimiento tales como las de Schumacher, Chapman-Richards, entre otras. Permite hallar el turno técnico óptimo o turno forestal óptimo.

2 Modelo económico: además de variables biológicoforestales, incluye variables de naturaleza económica (tales como costos de aprovechamiento de madera, precios, entre otras). Permite hallar el turno económico óptimo.
}

forestal (TVET). El presente estudio usa ambos enfoques o modelos, por ser los que se ajustan a la data de información disponible. El modelo TVAN se basa en el teorema de Fisher-Hotelling (F-H), mientras que el TVET utiliza el teorema de FaustmannPressler-Ohlin (F-P-O).

Debido a que la Normativa de Aprovechamiento Forestal de Bosques Húmedos Tropicales en Ecuador no fija el ciclo de tala correspondiente a la especie objeto de estudio, el presente trabajo tiene por objetivo general determinar los turnos óptimos, a través de maximizar y comparar la producción de beneficios económicos para el propietario o gestor forestal, empleando modelos que permiten obtener los períodos óptimos de tala, técnico (forestal) y económico, de romerillo blanco en Chinchipe, Ecuador. Para cumplir con tal propósito se utilizó información proveniente de 82 árboles de romerillo blanco registrados en 5 hectáreas de la Reserva Numbala, ubicada en la zona de amortiguamiento de la Reserva de la Biosfera Podocarpus-El Cóndor. Los objetivos específicos del presente trabajo son dos: (i) calcular y evaluar el turno técnicamente óptimo de la especie de romerillo blanco utilizando modelos de crecimiento forestal alternativos; (ii) estimar y evaluar -comparativamente- el turno económico óptimo utilizando los modelos de optimización económica de Fisher-Hotelling y Faustmann-Pressler-Ohlin.

\section{Materiales y métodos.}

El lugar de estudio se encuentra en la Reserva Numbala que es un bosque protegido, propiedad de la Fundación Conservacionista "Naturaleza \& Cultura Internacional" (NCI), que tiene sedes en Ecuador, Perú, México y Colombia. La reserva tiene uno de los mayores activos de capital natural forestal de dos especies de la familia Podocarpaceae: romerillo blanco y romerillo fino o colorado, y es uno de los últimos reductos con este tipo de bosque en la región andina. En el mapa 1 se muestra la ubicación de los bosques de romerillo blanco estudiados en la Reserva Numbala, Ecuador.

Cuando se trata del aprovechamiento de madera y el propósito es establecer el período óptimo de tala, se trabajan modelos forestales y económicos que permiten hallar el turno técnicamente óptimo y económicamente óptimo.

1 . Modelo forestal

La expresión que representa esta estimación es:

Dónde:

$$
V=f(t)(1)
$$

$V$ : volumen comercial de madera o masa forestal $\left(\mathrm{m}^{3} /\right.$ árbol o $\left.\mathrm{m}^{3} / \mathrm{ha}\right)$

$t$ : representación del tiempo o edad del árbol (años, por ejemplo) 


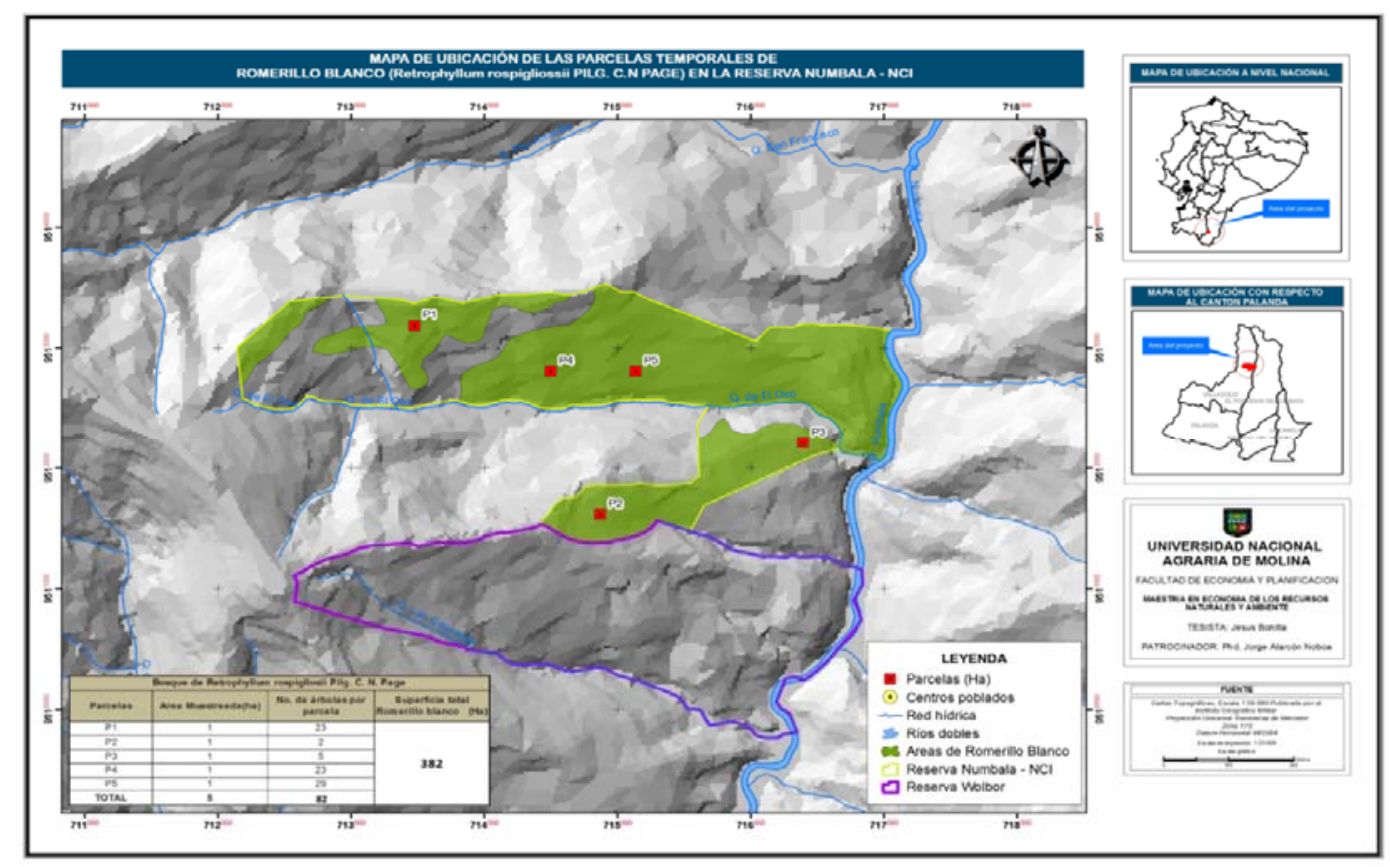

Mapa 1. Ubicación de los bosques de romerillo blanco estudiados en la Reserva Numbala, Ecuador.

$f(t)$ : Función de producción temporal o curva de crecimiento de volumen de madera.

Para determinar el turno forestal óptimo, o mejor momento de tala de un bosque, es preciso conocer el crecimiento absoluto de la especie forestal en términos de Crecimiento Medio Anual (CMA) e Incremento Corriente Anual (ICA), a lo largo del tiempo. El CMA es definido como el resultante del cociente entre el crecimiento periódico del volumen de madera y el número de años del período $[f(t) / t]$. El ICA es el cambio marginal anual de la función: $\mathrm{f}^{\prime}(t)$. Una propiedad importante que relaciona ambos crecimientos, es que $\mathrm{CMA}=\mathrm{ICA}$ cuando el crecimiento medio anual es máximo, condición que permite definir el turno técnico óptimo de tala $(t)$ en la gestión forestal (Riera et al., 2005). Para estimar los coeficientes de la relación funcional o ecuación (1), se requiere contar con el volumen comercial de madera y con la predicción de la edad del árbol " $t$ ", lo que se hace a partir de las ecuaciones (2) y (3) que se describen a continuación.

Finalmente, en el punto de la ecuación en que $\mathrm{CMA}=\mathrm{ICA}$, se determina el nivel óptimo de interés $t^{*}$ de tala (en años) y el volumen óptimo de madera, determinación que se consigue a partir de una de varias expresiones específicas como las representadas por las ecuaciones Cuadrática, Schumacher y Chapman-Richards (Tabla 1).

Cálculo del volumen comercial de madera sin corteza

El volumen comercial de madera sin corteza es la variable objetivo de la ecuación (1), es estimada con la siguiente fórmula:

$$
V_{i, t}=\left(\mathrm{DAP}^{2} \cdot \frac{\pi}{4}\right)-\left(H C_{i, t}\right)\left(F f_{i, t}\right)(2)
$$

Donde

$V$ : volumen sin corteza del árbol $i$ a la edad $t$, generalmente expresado en $\mathrm{m}^{3} / \mathrm{Ha}$.

DAP: Diámetro a la altura del pecho ${ }^{3}$ del árbol $i$ a la edad $t$, medido en $\mathrm{cm}$.

Tabla 1. Tipo de ecuaciones para la determinación de los turnos técnicamente óptimos, y volúmenes de madera a cosechar sosteniblemente por hectárea para el romerillo blanco.

\begin{tabular}{|c|c|c|}
\hline $\mathrm{N}^{\circ}$ & Ecuación & Funciones de crecimiento \\
\hline 1 & Ecuación Cuadrática & $V=f(t)=\beta_{0}+\beta_{1} t+\beta_{2} t^{2}$ \\
\hline 2 & Ecuación de Schumacher & $V=f(t)=\beta_{0} e^{-\beta_{1}\left(\frac{1}{t}\right)}$ \\
\hline 3 & Ecuación de Chapman-Richards & $V=f(t)=\beta_{0}\left(1-e^{-\beta_{1} t}\right)^{\beta_{2}}$ \\
\hline
\end{tabular}

${ }^{3} G=\left(\mathrm{DAP}^{2} \cdot \frac{\pi}{4}\right)$ es el área basal, representa un índice del grado de desarrollo de un bosque. Para un árbol individual se expresa en $\mathrm{m}^{2}$, y cuyas sumas por árbol determinan la densidad de un bosque $\left(\mathrm{m}^{2} / \mathrm{ha}\right)$. El área basal $(G)$ de un árbol se calcula como el área de un círculo de diámetro igual al DAP del árbol (Pereira et al., 1995). 
$H C$ : altura comercial del árbol $i$ en el tiempo $t$.

$\mathrm{F} f$ : factor de forma del árbol $i$ en el tiempo $t$.

Estimación de la edad del romerillo blanco

Para estimar la edad del árbol -que es la variable independiente de la ecuación (1)- se realizó un inventario forestal a nivel de muestreo estadístico y un estudio de las edades de los árboles de romerillo, usando la técnica de conteo de anillos de crecimiento (por dendrocronología). Se determinó así la edad real a partir de tarugos cilíndricos de madera ${ }^{4}$ para 22 árboles muestreados en campo con el uso del barreno de Pressler ${ }^{5}$ ). Para pronosticar la edad de la población de árboles registrados en el inventario forestal (82 árboles), se aplicó la función de edades del romerillo blanco mediante la siguiente expresión:

Donde

$$
t=f(\mathrm{DAP})(3)
$$

$t$ : edad del árbol; DAP: diámetro a la altura de pecho, en $\mathrm{cm}$.

La especificación funcional de la ecuación (3) usualmente se realiza mediante formas funcionales alternativas (destacan la función lineal, cuadrática, doble-logarítmica y semi-logarítmicas).

\section{Modelos económicos}

El turno económico óptimo suele definirse como la vida de la masa forestal que maximiza el valor actual neto (VAN) de la inversión subyacente. Considera una serie de supuestos como la existencia de un mercado perfecto del dinero, rendimiento y precios futuros -de madera e insumos-constantes, entre otros. Para calcular los óptimos de corte se usan dos modelos económicos: Fisher-Hotelling (Turno del Valor Presente Neto-TVPN) y Faustmann-Pressler-Ohlin (Turno del Valor de la Tierra Forestal-TVTF), que permiten maximizar los beneficios económicos del gestor forestal (Riera et al., 2005; Tietenberg \& Lewis, 2009; Romero, 1997). H):

Turno Económico Óptimo de Fisher-Hotelling (F-

Consiste en resolver la ecuación (4), con respecto al tiempo; en decir maximizar el Valor Actual Neto $(V A N)$ siguiente:

$$
V A N=p V(t) e^{-r t}-C(4)
$$

Donde

$p$ : precio de la madera

$V$ : volumen sin corteza del árbol, en función del tiempo " $t$ "

\footnotetext{
${ }^{4}$ Tarugo cilíndrico de madera: Porción de madera extraída de un árbol y con forma tubular.

5 Barreno de Pressler: herramienta que permite extraer muestras en forma de cilindro de madera para poder contar los anillos en el árbol y conocer su edad.
}

$r$ : la tasa social de descuento (de largo plazo para proyectos con especies de crecimiento lento)

$C$ : costos de plantado o gastos de inversión.

El uso de la condición necesaria, $\frac{d(V A N)}{d t}=0$, lleva a la relación F-H:

$$
\frac{V^{\prime}(t)}{V(t)}=r(5)
$$

En el nivel óptimo, el valor de " $\boldsymbol{t}$ " es aquel en que resulta indiferente cortar o no cortar la madera. Esta aproximación supone que el precio y costos son constantes a lo largo del tiempo.

Turno Económico Óptimo de Faustmann-PresslerOhlin (F-P-O):

En este paradigma se considera una cadena infinita de ciclos de plantación y, de una manera explícita, la existencia de un costo de oportunidad de la tierra. Conceptualiza el turno económico óptimo como aquél que maximiza la siguiente expresión del VET:

$$
V E T=\frac{p f(t) e^{-r t}-\sum_{j=1}^{t} K-\sum_{j=1}^{t} R}{1-e^{-r t}}(6)
$$

Donde:

VET: valor esperado de la Tierra.

$p$ : precio constante del recurso forestal.

$f(\mathrm{t})$ : volumen de madera cosechado en el período $" t "$.

$r$ : tasa de descuento (en este caso es la tasa social de descuento de largo plazo para proyectos forestales con especies de crecimiento lento, como romerillo blanco).

$K$ : gastos de aprovechamiento forestal.

$R$ : precio de alquiler de la tierra forestal.

Maximizando (6) se obtiene (con primera derivada igual a cero):

$$
p f^{\prime}(t)=r[p f(t)+S](7)
$$

Donde $S$ es el valor del suelo, obtenido por capitalización de una cadena infinita de inversiones. Nótese que para los turnos económicos (" $t$ ") se utilizan las formas funcionales forestales previas (Cuadrática, Shumacher y Chapman-Richards).

\section{Resultados y discusión.}

Para una ordenada presentación y discusión de resultados, es necesario mantener el orden del proceso metodológico. En tal sentido se presenta el cálculo de los indicadores y variables que llevan finalmente al uso de los modelos forestal y económico, para reportar los valores de turno técnico óptimo y el turno económico. 
Ecol. apl. Vol. 14 №2, pp. 127-137

Tabla 2. Volúmenes comerciales de Romerillo Blanco ${ }^{6}$

\begin{tabular}{|c|c|c|c|c|c|c|}
\hline \multirow[b]{3}{*}{ ESTADÍSTIC. } & \multicolumn{5}{|c|}{ PARCELAS (en Ha.) } & \multirow{3}{*}{$\begin{array}{c}\text { TOTAL } \\
\text { (5 Has) } \\
\text { VOL. } \\
\text { Comercial } \\
\text { (m³/Ha) }\end{array}$} \\
\hline & 1 & 2 & 3 & 4 & 5 & \\
\hline & $\begin{array}{c}\text { VOL. } \\
\text { Comercial }^{6} \\
\left(\mathbf{m}^{3} / \mathrm{Ha}\right)\end{array}$ & $\begin{array}{c}\text { VOL. } \\
\text { Comercia } \\
\text { l (m³/Ha) }\end{array}$ & $\begin{array}{c}\text { VOL. } \\
\text { Comercial } \\
\left(\mathbf{m}^{3} / \mathrm{Ha}\right)\end{array}$ & $\begin{array}{c}\text { VOL. } \\
\text { Comercial } \\
\left(\mathrm{m}^{3} / \mathrm{Ha}\right)\end{array}$ & $\begin{array}{c}\text { VOL. } \\
\text { Comercial } \\
\left(\mathbf{m}^{3} / \mathrm{Ha}\right)\end{array}$ & \\
\hline Mínimo & 0.94 & 10.33 & 0.65 & 0.42 & 0.00 & 12.34 \\
\hline Máximo & 26.71 & 19.66 & 43.12 & 19.05 & 29.76 & 138.29 \\
\hline Total & 193.13 & 29.99 & 76.97 & 180.73 & 219.73 & 700.55 \\
\hline Promedio & 8.40 & 14.99 & 15.39 & 7.86 & 7.58 & 54.22 \\
\hline Desv. Estandar & 5.46 & 6.60 & 19.03 & 5.35 & 6.32 & 42.76 \\
\hline $\mathrm{CV}(\%)$ & 64.97 & 44.00 & 123.64 & 68.07 & 83.43 & 384.12 \\
\hline
\end{tabular}

1. Determinación de los volúmenes comerciales de romerillo blanco

Para el cálculo de los volúmenes comerciales de los árboles de romerillo blanco, se usó el área basal (G), la altura comercial sin corteza (HC) y el factor de forma (Ff) (ver detalle de la Tabla A2 del Anexo). Los volúmenes comerciales promedio de madera sin corteza se presentan en la Tabla 2, a nivel de parcela muestreada, según la ecuación (2) anterior. En total se registró un volumen comercial sin corteza de 700.55 ${ }^{\mathrm{m} 3}$ en 5 Hay un volumen promedio de $140.1 \mathrm{~m}^{3} / \mathrm{ha}$.

2. Edades del romerillo blanco, a través de relación diámetro- edad

En base a la ecuación (3), se usaron -en este casocinco tipos de formas funcionales, luego estimadas por Mínimos Cuadrados Ordinarios- MCO, con la finalidad de pronosticar las edades promedio de romerillo blanco (en la población). Fueron 22 árboles muestreados en campo por el método dendrocronológico de conteo de anillos (barreno de Pressler). Según los criterios Akaike y Schwarz, la bondad de ajuste y la significancia estadística de los coeficientes, el modelo seleccionado fue el doble logarítmico (forma funcional III en la Tabla 3), expresando la posibilidad de tener la mejor predicción de las edades de los árboles en función del DAP.

En la Tabla 3 se aprecia que los coeficientes de la variable regresora de la ecuación (III) son significativos con 5\% de error. La bondad de ajuste es muy buena ( $\mathrm{R}^{2}$ ajustado de 0.97$)$. La ecuación de

${ }^{6}$ Los árboles Sequoia sempervirens del norte de California son frecuentemente citados como los árboles más grandes del mundo (se indica que alcanzan hasta $1203.4 \mathrm{~m} 3 / \mathrm{ha}$, y más de 3000 años de edad). No obstante que el volumen comercial de madera de bosques de romerillo es muy inferior a los bosques de Sequoia, los bosques de Romerillo en Numbala son el mayor bosque tropical en América del Sur, en términos de biomasa registrado en una Ha. de Bosque Natural. Los investigadores reportan volúmenes comerciales de 24.17 a $42 \mathrm{~m}^{3} /$ ha; esto muestra que son árboles muy grandes y con volúmenes importantes de madera comercial. Para efecto de este estudio se ha encontrado volúmenes mínimos -por parcela- de $12.34 \mathrm{~m}^{3} / 5$ Ha. y un máximo de $138.29 \mathrm{~m}^{3} / 5$ Ha. (Neill, D. 2012; Yaguana et al. 2012). regresión doble logarítmica (Log-Log), indica que la edad " $t$ " está directa y significativamente relacionada con el dap del romerillo blanco. Específicamente $\hat{\beta}_{1}$ indica que un aumento de 10 puntos porcentuales en el diámetro promedio de los árboles del bosque Numbala se asocia con un aumento de la edad de los árboles en $9 \%$. Cabe indicar que las edades reales de los romerillos blanco estuvieron entre de 68 a 398 años, mientras que las edades pronosticadas por la ecuación doble logarítmica están entre 4 y 427 años. Las edades pronosticadas para la población total se pueden evidenciar en la Tabla A1 del Anexo.

Tabla 3. Resultados de estimación MCO de las edades (t) de Romerillo Blanco

\begin{tabular}{|c|c|c|c|c|c|}
\hline $\begin{array}{c}\text { Variable } \\
\text { regresora } \\
\text { (DAP) }\end{array}$ & (I) & (II) & (III) & (IV) & (V) \\
\hline$\hat{\beta}_{0}$ & $\begin{array}{r}17.96 \\
(0.6) *\end{array}$ & $\begin{array}{r}4.04 \\
0.81\end{array}$ & 5.58 & 4.21 & 272.87 \\
\hline$\rho_{0}$ & $(0.06)^{*}$ & $(0.81)$ & $(0.00)$ & $(0.00)$ & $(0.00)$ \\
\hline$\hat{\beta}_{1}$ & 244.99 & 286.83 & 0.91 & 1.28 & 161.41 \\
\hline$\beta_{1}$ & $(0.00)$ & $(0.00)$ & $(0.00)$ & $(0.00)$ & $(0.00)$ \\
\hline$\hat{\beta}_{2}$ & & -24.19 & & & \\
\hline$P_{2}$ & & $(0.32)$ & & & \\
\hline $\mathrm{R}^{2}$ & 0.96 & 0.96 & 0.97 & 0.89 & 0.91 \\
\hline $\begin{array}{c}\mathrm{R}^{2} \\
\text { ajustado }\end{array}$ & 0.96 & 0.96 & 0.97 & 0.88 & 0.90 \\
\hline $\begin{array}{l}\text { Criterio } \\
\text { Akaike }\end{array}$ & 8.97 & 9.00 & -1.65 & -0.43 & 9.83 \\
\hline $\begin{array}{l}\text { Criterio } \\
\text { Schwarz }\end{array}$ & 9.07 & 9.15 & -1.55 & -0.33 & 9.93 \\
\hline
\end{tabular}

Notas: $\mathrm{MCO}=$ estimación por Mínimos Cuadrados Ordinarios; $\mathrm{DAP}=$ variable regresora que Significa diámetro a altura de pecho (entre paréntesis la Prob. asociada al estadístico " $t$ ").

Ecuaciones: lineal (I), cuadrática (II), doble-logarítmica (III), semilogarítmicas (IV y V).

Fuente: Elaboración propia

3. Turno de tala forestal de la especie Romerillo Blanco

Las tres funciones mostradas en la Tabla 1, correspondientes a la ecuación del modelo general (1), permitieron construir tres funciones de crecimiento para determinar el turno técnico óptimo de la especie en estudio, así como también la máxima cantidad de madera a extraer de manera sostenible (condición $\mathrm{CMA}=\mathrm{ICA}$ ). El CMA se obtuvo dividiendo el 
TURNOS TÉCNICO Y ECONÓMICO DE TALA PARA ARBOLES DE ROMERILLO EN ECUADOR Julio - Diciembre 2015

Tabla 4. Estimación del turno técnicamente óptimo

\begin{tabular}{|c|c|c|c|c|c|c|}
\hline \multirow{2}{*}{$\begin{array}{c}\text { Variable } \\
\text { dependiente: } \\
\text { Volumen de madera } \\
\text { Variable } \\
\text { independiente: Edad }\end{array}$} & \multicolumn{2}{|c|}{ Función Cuadrática } & \multicolumn{2}{|c|}{ Función Schumacher } & \multicolumn{2}{|c|}{$\begin{array}{l}\text { Función Chapman- } \\
\text { Richards }\end{array}$} \\
\hline & Estimadores & $\begin{array}{c}\text { Turno } \\
\text { Óptimo } \\
(t)\end{array}$ & Estimadores & $\begin{array}{c}\text { Turno } \\
\text { Óptimo } \\
(t)\end{array}$ & Estimadores & $\begin{array}{c}\text { Turno } \\
\text { Óptimo } \\
(t)\end{array}$ \\
\hline$\hat{\beta}_{0}$ & $\begin{array}{l}4.86 \\
(0.00)^{2}\end{array}$ & \multirow{8}{*}{76} & $\begin{array}{c}1.20 \\
(0.00)\end{array}$ & & $\begin{array}{c}5.04 \\
(0.00)\end{array}$ & \multirow{8}{*}{189} \\
\hline$\hat{\beta}_{1}$ & $\begin{array}{c}0.03 \\
(0.00)\end{array}$ & & $\begin{array}{c}-27.01 \\
(0.00)\end{array}$ & & $\begin{array}{r}-274.86 \\
(0.31)\end{array}$ & \\
\hline & 0.0003 & & & & 5.07 & \\
\hline$\beta_{2}$ & $(0.12)$ & & & & $(0.00)$ & \\
\hline $\mathrm{R}^{2}$ & 0.54 & & 0.23 & 77 & 0.12 & \\
\hline $\mathrm{R}^{2}$ ajustado & 0.53 & & 0.22 & & 0.09 & \\
\hline Criterio Akaike & 6.11 & & 3.46 & & 6.07 & \\
\hline Criterio Schwarz & 6.20 & & 3.52 & & 6.16 & \\
\hline $\begin{array}{l}{ }^{2} \text { Los valores en parér } \\
\mathrm{V}=\text { Volumen comerc } \\
\hat{\beta}_{1}=\text { coeficiente asoc } \\
\hat{\beta}_{2}=\text { coeficiente asoc } \\
\text { TO= Óptimo técnico } \\
\text { Fuente: Elaboración }\end{array}$ & $\begin{array}{l}\text { is represent } \\
\text { de madera s } \\
\text { o a edad (ed } \\
\text { o a edad, en } \\
\text { la edad de t } \\
\text { oia }\end{array}$ & $\begin{array}{l}\text { las proba } \\
\text { orteza d } \\
\text { del árbol } \\
\text { ma cuad } \\
\text { para el ro }\end{array}$ & $\begin{array}{l}\text { lidades asoc } \\
\text { romerillo bl } \\
\text { e romerillo } \\
\text { tica o expon } \\
\text { erillo blanc }\end{array}$ & $\begin{array}{l}\text { das al es } \\
\text { co en " } t \text { ' } \\
\text { inco en a } \\
\text { cial. }\end{array}$ & $\begin{array}{l}\text { lístico “ } t \text { " (p } \\
\text { ños. } \\
\text { os). }\end{array}$ & \\
\hline
\end{tabular}

sostenible de madera de romerillo blanco es cosechar un máximo de 65.25 $\mathrm{m}^{3} / \mathrm{Ha}$, de los $140.11 \quad \mathrm{~m}^{3} / \mathrm{Ha}$ disponibles, respetando un ciclo de tala mínimo de 77 años. La Tabla 5 muestra los diferentes niveles de extracción sustentable de madera, siendo el modelo óptimo de Schumacher el que reporta un aprovechamiento de los volúmenes comerciales $\quad \sin$ corteza de 65.25

crecimiento periódico del volumen de madera y el número de años del período $\left(\mathrm{m}^{3} / \mathrm{Arb}\right.$.), mientras que el ICA se determinó evaluando la condición de primer orden en un punto para cada modelo funcional de crecimiento.

En la Tabla 4 se presentan los resultados de las tres estimaciones antes indicadas. La función Schumacher fue finalmente seleccionada como la mejor, en base a los menores valores de los criterios Akaike y Schwarz, la bondad de ajuste y la significancia de los estimadores. La función Schumacher permitió identificar el turno óptimo técnico en el periodo " $t$ "; también el rendimiento óptimo de producción de madera comercial sin corteza para la Reserva Numbala.

Luego de determinar el periodo de rotación forestal se analizó la sustentabilidad del "romerillo blanco" en el bosque natural de la Reserva Numbala, asumiendo que el óptimo técnico de tala de dichos modelos determina el comportamiento sostenible del aprovechamiento de la madera. Cabe recordar que el óptimo técnico busca maximizar la cantidad de madera que se obtiene por unidad de tiempo. Para determinar el aprovechamiento sostenible puede hacerse uso de la edad total del bosque maduro o la edad óptima de tala estimada en cada ecuación. Estimando el aprovechamiento sostenible, e introduciendo los valores de la Tabla 4 en la ecuación Schumacher estimada $\quad[\widehat{V}=f(t)=$ $\left.1.203398 e^{-27.00519\left(\frac{1}{77}\right)}\right]$, se obtiene como resultado un aprovechamiento sostenible (en volumen de madera) de $0.8474 \mathrm{~m}^{3} / \mathrm{Ha} /$ año; si este valor multiplica por el óptimo técnico estimado en 77 años, el resultado será de $65.25 \mathrm{~m}^{3} / \mathrm{Ha}$. Lo anterior significa que la condición optima de aprovechamiento $\mathrm{m}^{3} / \mathrm{Ha}$, y un stock de capital natural forestal a mantenerse de $74.86 \mathrm{~m}^{3} / \mathrm{Ha}$.

4. Turno económico óptimo utilizando modelos Económicos

Para obtener el turno económico óptimo se usaron los modelos de Fisher-Hotelling (F-H) y el de Faustmann-Pressler-Ohlin (F-P-O). En ambos casos las variables consideradas en la estimación fueron: volumen comercial de madera sin corteza, edad del árbol, precios de madera constantes, gastos anuales de explotación constantes, ingresos brutos ${ }^{7}$, beneficios netos y tasas de descuento. Las tasas de descuentos utilizadas o recomendadas en proyectos ambientales de largo plazo, para un recurso forestal de lento crecimiento, como es el caso del romerillo blanco fueron: $0.33 \%, 0.52 \%, 0.75 \%$, y $1 \%$ (Edwars, 2002; Restrepo \& Alviar, 2010; Alvares \& Koskelab, 2006; Azqueta et al, 2007; Weitzman, 2001; Correa, 2006; Correa 2008).

Enfoque Fisher- Hotteling (F-H)

En este caso, la estimación del turno económico forestal del bosque de romerillo blanco consistió en definir el máximo del Valor Presente Neto (VPN) de la inversión privada. El método consideró también una serie de supuestos: mercado perfecto del dinero, precios futuros constantes (tanto de madera como de insumos utilizados), rendimientos madereros constantes (para un nivel dado de insumo), entre otros. La Tabla 6 muestra los resultados del nivel de extracción sustentable de madera, los turnos forestales, turnos económicos óptimos por Fisher-

\footnotetext{
${ }^{7} \mathrm{La}$ moneda actual en Ecuador es el dólar de los Estados Unidos de América, que en el año 2000 sustituyó al sucre, anterior divisa oficial. Sin embargo se emiten billetes propios de 100, 50, 20, 10, 5, 2 y 1 dólar; también monedas.
} 
Tabla 5. Funciones de crecimiento indicativos del aprovechamiento sustentable del recurso forestal

\begin{tabular}{|c|c|c|c|c|c|c|c|}
\hline \multirow[b]{2}{*}{$\begin{array}{l}\text { Forma } \\
\text { funcional }\end{array}$} & \multirow{2}{*}{$\begin{array}{c}\text { Óptimo } \\
\text { técnico } \\
\text { de tala } \\
(t)\end{array}$} & \multirow{2}{*}{$\begin{array}{c}\text { Capital } \\
\text { físico } \\
\text { natural } \\
\text { forestal }^{8} \\
\text { (madera, } \\
\left.\mathrm{m}^{3} / \mathrm{Ha}\right)\end{array}$} & \multirow{2}{*}{$\begin{array}{c}\text { Stock } \\
\text { aprove- } \\
\text { chable de } \\
\text { madera } \\
\text { sustentable } \\
(\mathrm{Sams})^{9} \text {, } \\
\left(\mathrm{m}^{3} / \mathrm{Ha}\right)\end{array}$} & \multirow{2}{*}{$\begin{array}{c}\text { Capital } \\
\text { natural } \\
\text { forestal } \\
\text { remanente } \\
(\mathrm{Cnfr})^{10} \\
\left(\mathrm{~m}^{3} / \mathrm{Ha}\right)\end{array}$} & \multicolumn{3}{|c|}{$\begin{array}{c}\% \text { de aprovechamiento para sustentabilidad } \\
\text { del recurso forestal }\end{array}$} \\
\hline & & & & & $\begin{array}{c}\% \text { Madera } \\
\text { aprovechable } \\
(\% \mathrm{Ma})^{\mathbf{1 1}}\end{array}$ & $\begin{array}{c}\% \text { Stock } \\
\text { sustentable } \\
(\% \mathrm{Ss})\end{array}$ & $\begin{array}{l}\% \text { Stock } \\
\text { total forestal } \\
(\% \text { Stf })\end{array}$ \\
\hline$\overline{\mathrm{FC}}$ & 76 & 140.11 & 44.41 & 95.70 & 31.7 & 68.3 & 100 \\
\hline FSch & 77 & 140.11 & 65.25 & 74.86 & 46.57 & 53.43 & 100 \\
\hline FR-Ch & 189 & 140.11 & 82.73 & 57.38 & 59.05 & 40.95 & 100 \\
\hline
\end{tabular}

Funciones forestales: $\mathrm{FC}=$ Función Cuadrática, $\mathrm{FSCh}=$ Función de Schumacher,

FR-Ch= Función de Chapman-Richards.

Fuente: Elaboración propia

Hotelling y el valor presente neto que maximiza la inversión económica del gestor forestal.

En la Tabla 6 puede notarse que para el óptimo de Fisher-Hotteling, la condición de equilibrio (o punto de indiferencia) para tasas de descuento del 0.33 , $0.52 \%, 0.75 \%$ y $1.0 \%$, se alcanzó en $304,193,134$ y 100 años respectivamente. Es importante destacar que el indicador de la condición F-H permitió obtener turnos económicos-óptimos más largos que el óptimo técnico de 77 años (caso de la Función ajustada de Schumacher).

Enfoque Faustmann-Pressler-Ohlin (F-P-O)

El turno económico forestal a partir del modelo Faustmann-Pressler-Ohlin consistió en definir e incluir el Valor de la Tierra Forestal (VET).Este método también consideró los mismos supuestos anteriores (mercado perfecto de dinero, precios y costos futuros constantes, rendimientos madereros constantes, entre otros) más el supuesto que el suelo forestal se puede comprar, vender y arrendar en condiciones competitivas. Asimismo, se usaron tasas de descuento para proyectos forestales de largo plazo del $0.33,0.52,0.75$, y $1.0 \%$ (Tabla 7 ).

Siguiendo el orden de los modelos presentados en la Tabla 7, se puede ver que para el óptimo de F-P-O, la condición de equilibrio para tasas de descuento del $0.33,0.52 \%, 0.75 \%$ y $1.0 \%$, se alcanzó en 208,132 , 92 y 69 años respectivamente. El indicador de la condición F-P-O (considerado un indicador completo, por contener el valor de la tierra forestal) permitió obtener turnos económicos-óptimos generalmente menos largos que el óptimo económico de FisherHotelling (F-H). Es importante considerar las diferencias entre el periodo económico de tala obtenido y el turno técnico óptimo (Tabla 4), puesto que este último contiene el mayor volumen comercial de madera por unidad de tiempo. Considerando el turno óptimo según la función de Schumacher, se aprecia de manera general que, para romerillo blanco, la relación entre los óptimos calculados es la siguiente:

\section{T-FPO $<$ T-Op.F $<$ T-FH}

Por tanto, la evidencia empírica nos dice que si estamos analizando una especie de crecimiento lento y largo (como romerillo blanco), al incrementarse la tasa de descuento en el modelo económico, el turno forestal disminuye (tasas de descuento más bajas producen turnos más largos). Por otro lado, al trabajar con especies de crecimiento rápido (menos de 25 años), es muy probable que se usen tasas de descuento superiores a las usadas con especies de crecimiento lento; esto se debe principalmente a que los rendimientos madereros acumulados en el primer caso son más veloces que en el segundo, y, por lo tanto, los proyectos forestales para especies de rápido crecimiento, son más financiables por entidades crediticias. Finalmente es importante añadir que los proyectos forestales de reforestación de especies nativas, con fines comerciales, no son financiables por los acreedores, debido al tiempo de espera para la cosecha (turnos muy largos); por tanto, si se quiere lograr la sustentabilidad del recurso forestal aprovechado, a más de considerar y respetar los ciclos económicos de tala calculados, se debe tomar en cuenta que financiar proyectos de reforestación con especies nativas a bajas tasas de descuento y en muy largo plazo, puede garantizar cadenas sucesivas de aprovechamiento forestal y evitar así la degradación y agotamiento futuro del recurso.

\footnotetext{
${ }^{8}$ Capital físico natural: todo stock natural que genera un flujo de bienes y servicios útiles o renta natural a lo largo del tiempo; pueden provenir de la explotación de recursos forestales, minerales, pesqueros, etc. (Costanza y Daly, 1992; Riera et al. 2005; Azqueta et al. 2007). En este caso el capital físico natural forestal comprende los volúmenes comerciales de madera promedios $\left(\mathrm{m}^{3} / \mathrm{ha}\right)$, en pie, registrados en el inventario forestal realizado en los bosques de la Reserva Numbala.

9 Stock aprovechable de madera sustentable: cantidad de madera aprovechable sosteniblemente por hectárea.

${ }^{10} \mathrm{El}$ stock total de madera comercial (Stmc) es de 140.11 $\mathrm{m}^{3} /$ ha. Stmc - Sams $=$ Cnfr.

$11 \%$ de madera aprovechable $(\mathrm{Ma}=\mathrm{Stmc}-\mathrm{Cnfr})$. Ma, Ss y

Stf se expresan en valores porcentuales.
} 
Tabla 6. Variación de turnos óptimos y valor presente neto que maximiza la inversión de un "stand" de romerillo blanco, según condición de Fisher-Hotteling (precios constantes).

\begin{tabular}{|c|c|c|c|c|c|c|}
\hline \multirow{3}{*}{$\begin{array}{l}\text { Funciones } \\
\text { bio- } \\
\text { Forestales }\end{array}$} & \multirow{3}{*}{$\begin{array}{l}\text { VCMsc } \\
(\mathrm{m} 3 / \mathrm{Ha})\end{array}$} & \multirow{3}{*}{$\begin{array}{l}\text { TOp.F } \\
(t)\end{array}$} & \multicolumn{4}{|c|}{$\begin{array}{l}\text { Turno por condición Fisher Hotteling } \\
\text { TOp.E }(t)\end{array}$} \\
\hline & & & $\mathrm{r}_{1}$ & $\mathrm{r}_{2}$ & $\mathrm{r}_{3}$ & $\mathrm{r}_{4}$ \\
\hline & & & $0.33 \%$ & $0.52 \%$ & $0.75 \%$ & $1 \%$ \\
\hline $\mathrm{FC}$ & 44.41 & 76 & $\begin{array}{c}303 \\
(2051.16)^{*}\end{array}$ & $\begin{array}{c}193 \\
(1302.93)\end{array}$ & $\begin{array}{l}134 \\
(904.40)\end{array}$ & $\begin{array}{l}100 \\
(679.13)\end{array}$ \\
\hline FSCh & 65.25 & 77 & $\begin{array}{l}304 \\
(3516.05)\end{array}$ & $\begin{array}{l}193 \\
(2233.45)\end{array}$ & $\begin{array}{c}134 \\
(1550.30)\end{array}$ & $\begin{array}{c}101 \\
(1164.16)\end{array}$ \\
\hline FR-Ch & 82.73 & 189 & $\begin{array}{l}303 \\
(3516.05)\end{array}$ & $\begin{array}{l}193 \\
(3013.95)\end{array}$ & $\begin{array}{c}134 \\
(2092.06)\end{array}$ & $\begin{array}{l}100 \\
(157098)\end{array}$ \\
\hline
\end{tabular}

Funciones forestales: FC: Función Cuadrática; FSCh: Función de Schumacher; FR-Ch: Función Chapman-Richards. VCMsc: Volumen comercial de madera sin corteza para romerillo blanco $\left(\mathrm{m}^{3}\right.$ por $\left.\mathrm{Ha}\right)$

TOp.F $(t)$ : Turno forestal técnicamente óptimo en el período " $t$ ".

TOp.E $(t)$ : Turno económico óptimo en " $t$ " años por la condición de F-H.

$\mathrm{r}_{1}, \mathrm{r}_{2}, \mathrm{r}_{3}, \mathrm{r}_{4}$ : Tasas de descuentos intertemporales.

*Los valores en paréntesis representan la maximización de beneficios económicos (ingresos obtenidos del gestor) en valor presente neto $(\$ / \mathrm{Ha})$.

Fuente: Elaboración propia

\section{Conclusiones.}

1. El stock forestal comercial de madera total bruta (sin corteza) en 5 hectáreas muestreadas de romerillo blanco fue de $701 \mathrm{~m}^{3}$ con un promedio de 140.1 $\mathrm{m}^{3} / \mathrm{Ha}$. Las edades calculadas de la muestra $(22$ árboles), con el barreno de Pressler, son un indicador de que el bosque natural de romerillo blanco es un bosque en estado maduro. Asimismo, el volumen comercial de madera sin corteza y las edades pronosticadas de la población total de árboles $(82$ árboles de romerillo blanco), en función del diámetro a la "altura de pecho", ayudaron a explicar los turnos óptimos de aprovechamiento para la especie en estudio.

2. La Normativa Ecuatoriana de Manejo Forestal Sustentable para aprovechamiento de madera en bosque húmedo, no fija ciclos de tala para romerillo blanco; de allí la importancia de los resultados obtenidos en este estudio. El valor óptimo técnico de tala, desde la ciencia forestal, encontrado mediante uso de un modelo funcional Schumacher, corresponde a 77 años. Entonces, para propiciar un nuevo aprovechamiento forestal en la misma hectárea, a tal edad debería cosecharse $65.25 \mathrm{~m}^{3} / \mathrm{Ha}$, y dejarse 74.86 $\mathrm{m}^{3} /$ Ha para que cada 77 años vuelvan a crecer a un total de $140.11 \mathrm{~m}^{3} / \mathrm{Ha}$. De esta forma el crecimiento forestal sería sustentable, pues se evitaría reducir el stock del recurso forestal por la sobreexplotación incontrolada, generalmente originada por el gestor forestal; sin embargo tales óptimos forestales no constituyen necesariamente valores óptimos desde el punto de vista económico.

3. Las edades óptimas de tala, de compatibilidad entre los óptimos forestal y económico, resultaron adecuadas con una tasa de descuento de $1 \%$. Para tal circunstancia, según la función óptima de Schumacher, las edades óptimas de tala para el mismo stand de bosque $(1 \mathrm{Ha})$, resultaron ser variantes. Según el modelo Faustmann-Pressler-Ohlin, el turno

Tabla 7. Variación de turnos óptimos y valor presente neto que maximiza la inversión de un "stand" de romerillo blanco, según condición Faustmann-Pressler-Ohlin (F-P-O).

\begin{tabular}{|c|c|c|c|c|c|c|}
\hline \multirow{2}{*}{$\begin{array}{l}\text { Funciones } \\
\text { bio- } \\
\text { Forestales }\end{array}$} & \multirow{2}{*}{$\begin{array}{l}\text { VCMsc } \\
(\mathrm{m} 3 / \mathrm{Ha})\end{array}$} & \multirow{2}{*}{$\begin{array}{l}\text { TOp.F } \\
(t)\end{array}$} & \multicolumn{4}{|c|}{$\begin{array}{c}\text { Turno por condición FPO } \\
\text { TOp.E }(t)\end{array}$} \\
\hline & & & $\begin{array}{c}\mathrm{r}_{1} \\
0.33 \%\end{array}$ & $\begin{array}{c}\mathrm{r}_{2} \\
0.52 \%\end{array}$ & $\begin{array}{c}\mathrm{r}_{3} \\
0.75 \%\end{array}$ & $\begin{array}{c}\mathrm{r}_{4} \\
1.0 \%\end{array}$ \\
\hline FPC & 44.41 & 76 & $\begin{array}{l}103 \\
(453.60)^{*}\end{array}$ & $\begin{array}{c}65 \\
(262.57)\end{array}$ & $\begin{array}{c}45 \\
(160.80)\end{array}$ & $\begin{array}{c}34 \\
(103.30)\end{array}$ \\
\hline FSCh & 65.25 & 77 & $\begin{array}{c}208 \\
(4295.32)\end{array}$ & $\begin{array}{c}132 \\
(2700.59)\end{array}$ & $\begin{array}{c}92 \\
(1851.20)\end{array}$ & $\begin{array}{c}69 \\
(1371.06)\end{array}$ \\
\hline FR-Ch & 82.73 & 189 & $\begin{array}{c}305 \\
(4718.35) \\
\end{array}$ & $\begin{array}{c}195 \\
(2987.57) \\
\end{array}$ & $\begin{array}{c}136 \\
(2065.73) \\
\end{array}$ & $\begin{array}{c}102 \\
(1544.73) \\
\end{array}$ \\
\hline
\end{tabular}

Funciones forestales: FC: Función Cuadrática; FSCh: Función de Schumacher; FR-Ch: Función Chapman-Richards.

VCMsc: Volumen comercial de madera sin corteza para romerillo blanco $\left(\mathrm{m}^{3}\right.$ por $\left.\mathrm{Ha}\right)$

TOp.F ( $t$ : : Turno forestal técnicamente óptimo en el período " $t$ ".

TOp.E $(t)$ : Turno económico óptimo en " $t$ " años por la condición de F-P-O.

$\mathrm{r}_{1}, \mathrm{r}_{2}, \mathrm{r}_{3}, \mathrm{r}_{4}$ : Tasas de descuentos intertemporales.

*Los valores en paréntesis representan la maximización de beneficios económicos (ingresos obtenidos del gestor) en valor presente neto $(\$ / \mathrm{Ha})$.

Fuente: Elaboración propia 
Ecol. apl. Vol. 14 №2, pp. 127-137

económico óptimo fue de 69 años, mientras que en el caso del modelo Fisher-Hotteling fue de 101 años. En conclusión, los modelos económicos de F-P-O y F-H, con una tasa de descuento de $1 \%$, resultaron ser apropiados para determinar la edad de corte del romerillo blanco, abarcando un período económico óptimo de tala entre 69 y 101 años, con un intervalo de valores netos actualizados entre 1371.1 \$/Ha y 1164.2 \$/Ha. Tal intervalo óptimo incluye el turno técnico óptimo obtenido (77 años), comprendiendo buenos indicadores límites de la edad de tala económica óptima.

\section{Agradecimientos.}

Los autores agradecen a los funcionarios de la Reserva Numbala y a la organización "Naturaleza y Cultura Internacional"- NCI por el apoyo logístico para la realización del trabajo de campo. También a los profesionales Juan Magallanes, Ramón Diez, Agapito Linares (UNALM), Oswaldo Ganzhi por una lectura preliminar del documento y a tres revisores anónimos por muy buenas recomendaciones en la etapa final de elaboración del mismo.

\section{Literatura citada.}

Álvarez H. \& Koskelab E. 2006. Does risk aversion accelerate optimal forest rotation under uncertainty? En: Journal of forest economic 12 (2006): 171-184.

Azqueta D., Alviar M., Domínguez L. \& O'Ryan R. 2007.Introducción a la economía ambiental. Mc Graw Hill Ed. Madrid (p. 99-211).

Bonilla J. 2005. Proyecto implementación del plan de manejo de la subcuenca Numbala: Plan de manejo eco turístico participativo comunitario de los Helechos y la Esmeralda pertenecientes a la subcuenca Numbala, Ecuador.

Correa F. 2006. La tasa social de descuento y el medio ambiente. En: Revista Lecturas de Economía, 64 (enerojunio), pp. 91-116. Universidad de Antioquia. Colombia.

Correa F. 2008. El debate de la tasa social de descuento de largo plazo: estado de arte. En: Revista Equidad y Desarrollo $\mathrm{N}^{\circ}$ 10: 61-82 (Julio-Diciembre).

Costanza R. \& Daly H. 1992. Natural Capital and Sustainable Development. Conservation Biology 6: 3746.
Neill D. 1981. Numbala, el último refugio de los podocarpus gigantes. Loja, Ecuador. 2 pp.

Edwards E. 2002. La tasa de descuento en proyectos de largo plazo. Pontificia Universidad Católica de Chile. Instituto de Economía. Paper $\mathrm{N}^{\circ}$ 231. Santiago, Chile. $29 \mathrm{pp}$.

Gálvez J., Aguirre Z., Sánchez O. \& López N. 2003. Estado actual de conservación y posibilidades de manejo del romerillo en la región suroccidental del Parque Nacional Podocarpus. Universidad Técnica Particular de Loja\& Ministerio del Ambiente, Ecuador.

Lojan L. 1992. El verdor de los Andes: árboles y arbustos nativos para el desarrollo forestal alto andino. FAO, proyecto de desarrollo forestal participativo en los Andes-Quito, Ecuador.

PREDESUR. 1975. Inventario forestal y aprovechamiento de los bosques del sur. Loja, Ecuador. Ministerio de Agricultura, $209 \mathrm{pp}$.

Pereira C., Padilla S., Aguirre, Z. \& Mansur E. 1995. Proyecto Regional FAO. Desarrollo Forestal Participativo en Los Andes. Quito.

Restrepo C. \& Alviar M. 2010. Tasa de descuento y rotación forestal: El Caso del Eucalyptus Saligna. Lecturas de economía. No. 73. Medellín, Colombia. pp. 149-164.

Riera P., García D., Kristrom B. \& Brannlund R. 2005. Manual de Economía Ambiental y de los Recursos Naturales. Editorial Thompson. España.

Rojas A. 2005. Pinos colombianos: ocho nativos en peligro. Artículo en revista "El Mueble y la Madera". Colombia. pp. 18-25.

Romero C. 1997. Economía de los recursos ambientales y naturales. Ed. Alianza Economía, España.

Tietenberg T. \& Lewis L. 2009.Environmental and Natural Resources Economics. Pearson Editores, 2a edición. Reading, MA, USA.

Trujillo E. 2007. Guía de Reforestación. Pino romerón: Retrophyllum rospigliossi (Pilg.) C. N Page. Primera edición. Diseño e impresión DAYBER. Bogotá, Colombia.

Yaguana C., Lozano D., Neill D. \& Asanza M. 2012. Diversidad florística y estructura del bosque nublado del Río Numbala, Zamora-Chinchipe, Ecuador: el "bosque gigante" de Podocarpaceae adyacente al Parque Podocarpus. En Revista Amazónica: Ciencia y Tecnología, 1(3): 226-247.

Weitzman M. 2001.Gamma Discounting. En: American Economic Review 91.1: 261-271. 
TURNOS TÉCNICO Y ECONÓMICO DE TALA PARA ARBOLES DE ROMERILLO EN ECUADOR Julio - Diciembre 2015

\section{ANEXO}

Tabla A1. Edades pronosticadas por el modelo Log-Log para el romerillo

\begin{tabular}{|c|c|c|}
\hline N0. DE ÁRBOLES & DAP (M) & EDAD_PRED (t) \\
\hline 1 & 0,01 & 4 \\
\hline 2 & 0,15 & 47 \\
\hline 3 & 0,20 & 60 \\
\hline 4 & 0,22 & 67 \\
\hline 5 & 0,25 & 74 \\
\hline 6 & 0,25 & 75 \\
\hline 7 & 0,25 & 76 \\
\hline 8 & 0,26 & 77 \\
\hline 9 & 0,29 & 87 \\
\hline 10 & 0,30 & 87 \\
\hline 11 & 0,30 & 87 \\
\hline 12 & 0,31 & 92 \\
\hline 13 & 0,33 & 97 \\
\hline 14 & 0,35 & 101 \\
\hline 15 & 0,35 & 102 \\
\hline 16 & 0,35 & 103 \\
\hline 17 & 0,36 & 103 \\
\hline 18 & 0,37 & 106 \\
\hline 19 & 0,40 & 114 \\
\hline 20 & 0,49 & 137 \\
\hline 21 & 0,57 & 157 \\
\hline 22 & 0,57 & 159 \\
\hline 23 & 0,63 & 173 \\
\hline 24 & 0,64 & 175 \\
\hline 25 & 0,64 & 177 \\
\hline 26 & 0,65 & 179 \\
\hline 27 & 0,67 & 185 \\
\hline 28 & 0,69 & 189 \\
\hline 29 & 0,69 & 189 \\
\hline 30 & 0,69 & 189 \\
\hline 31 & 0,71 & 193 \\
\hline 32 & 0,72 & 196 \\
\hline 33 & 0,73 & 198 \\
\hline 34 & 0,74 & 199 \\
\hline 35 & 0,75 & 202 \\
\hline 36 & 0,76 & 207 \\
\hline 37 & 0,79 & 212 \\
\hline 38 & 0,79 & 213 \\
\hline 39 & 0,79 & 213 \\
\hline 40 & 0,79 & 213 \\
\hline 41 & 0,81 & 217 \\
\hline
\end{tabular}

\begin{tabular}{|c|c|c|}
\hline 42 & 0,81 & 218 \\
\hline 43 & 0,82 & 221 \\
\hline 44 & 0,82 & 221 \\
\hline 45 & 0,84 & 224 \\
\hline 46 & 0,84 & 225 \\
\hline 47 & 0,85 & 228 \\
\hline 48 & 0,85 & 228 \\
\hline 49 & 0,86 & 231 \\
\hline 50 & 0,87 & 233 \\
\hline 51 & 0,87 & 233 \\
\hline 52 & 0,88 & 236 \\
\hline 53 & 0,88 & 236 \\
\hline 54 & 0,89 & 237 \\
\hline 55 & 0,90 & 239 \\
\hline 56 & 0,90 & 241 \\
\hline 57 & 0,90 & 240 \\
\hline 58 & 0,91 & 242 \\
\hline 59 & 0,91 & 242 \\
\hline 60 & 0,92 & 244 \\
\hline 61 & 0,92 & 245 \\
\hline 62 & 0,93 & 247 \\
\hline 63 & 0,94 & 250 \\
\hline 64 & 0,95 & 252 \\
\hline 65 & 0,95 & 253 \\
\hline 66 & 0,96 & 253 \\
\hline 67 & 0,96 & 254 \\
\hline 68 & 0,98 & 259 \\
\hline 69 & 1,00 & 263 \\
\hline 70 & 1,01 & 265 \\
\hline 71 & 1,01 & 267 \\
\hline 72 & 1,01 & 267 \\
\hline 73 & 1,03 & 271 \\
\hline 74 & 1,04 & 273 \\
\hline 75 & 1,06 & 278 \\
\hline 76 & 1,08 & 282 \\
\hline 77 & 1,13 & 296 \\
\hline 78 & 1,17 & 305 \\
\hline 79 & 1,37 & 350 \\
\hline 80 & 1,38 & 354 \\
\hline 81 & 1,38 & 355 \\
\hline 82 & 1,70 & 427 \\
\hline
\end{tabular}

Fuente: elaboración propia 
Tabla A2. Estimación de los volúmenes comerciales sin corteza para el romerillo blanco en Numbala- Ecuador (presentación de muestra de datos de 31 árboles, de un total de 82).

\begin{tabular}{|c|c|c|c|c|c|c|c|c|c|c|}
\hline $\begin{array}{c}\text { NO. } \\
\text { ARBOL }\end{array}$ & $\begin{array}{c}\text { EDAD } \\
\text { (AÑ) }\end{array}$ & $\begin{array}{c}\text { DAPcc } \\
\text { (M) }\end{array}$ & $\begin{array}{c}\text { DAPsc } \\
\text { (M) }\end{array}$ & $\begin{array}{c}\text { Gcc(M/2/5 } \\
\text { HA) }\end{array}$ & $\begin{array}{c}\text { Gsc (M/2 } / 5 \\
\text { HA) }\end{array}$ & HC (M) & HT (M) & 'FF & $\begin{array}{c}\text { VOL. COM. } \\
\text { (M/5HA) }\end{array}$ & $\begin{array}{c}\text { VOL. TOTAL } \\
\text { (M/3HA) }\end{array}$ \\
\hline 1 & 199 & 0,97 & 0,90 & 0,75 & 0,64 & 28 & 44 & 0,76 & 13,66 & 24,92 \\
\hline 2 & 106 & 0,44 & 0,37 & 0,15 & 0,11 & 23 & 31 & 0,76 & 1,84 & 3,52 \\
\hline 3 & 190 & 0,91 & 0,84 & 0,65 & 0,55 & 25 & 35 & 0,76 & 10,54 & 17,31 \\
\hline 4 & 266 & 1,44 & 1,37 & 1,62 & 1,46 & 24 & 36 & 0,76 & 26,71 & 44,29 \\
\hline 5 & 193 & 0,93 & 0,86 & 0,68 & 0,58 & 24 & 30 & 0,76 & 10,66 & 15,58 \\
\hline 6 & 181 & 0,86 & 0,79 & 0,58 & 0,49 & 26 & 35 & 0,76 & 9,67 & 15,43 \\
\hline 7 & 185 & 0,88 & 0,81 & 0,61 & 0,52 & 23 & 35 & 0,76 & 9,05 & 16,24 \\
\hline 8 & 166 & 0,76 & 0,69 & 0,46 & 0,38 & 30 & 40 & 0,76 & 8,62 & 13,93 \\
\hline 9 & 163 & 0,74 & 0,67 & 0,44 & 0,36 & 18 & 27 & 0,76 & 4,89 & 8,94 \\
\hline 10 & 200 & 0,98 & 0,91 & 0,75 & 0,65 & 18 & 24 & 0,76 & 8,91 & 13,77 \\
\hline 11 & 104 & 0,43 & 0,36 & 0,14 & 0,10 & 18 & 22 & 0,76 & 1,37 & 2,39 \\
\hline 12 & 198 & 0,97 & 0,90 & 0,74 & 0,63 & 18 & 28 & 0,76 & 8,66 & 15,65 \\
\hline 13 & 172 & 0,80 & 0,73 & 0,50 & 0,42 & 24 & 32 & 0,76 & 7,61 & 12,19 \\
\hline 14 & 165 & 0,76 & 0,69 & 0,45 & 0,37 & 22 & 30 & 0,76 & 6,27 & 10,36 \\
\hline 15 & 91 & 0,37 & 0,30 & 0,11 & 0,07 & 18 & 28 & 0,76 & 0,94 & 2,24 \\
\hline 16 & 192 & 0,92 & 0,85 & 0,67 & 0,57 & 24 & 36 & 0,76 & 10,43 & 18,31 \\
\hline 17 & 186 & 0,89 & 0,82 & 0,62 & 0,53 & 24 & 35 & 0,76 & 9,66 & 16,60 \\
\hline 18 & 199 & 0,97 & 0,90 & 0,74 & 0,64 & 18 & 28 & 0,76 & 8,72 & 15,75 \\
\hline 19 & 216 & 1,08 & 1,01 & 0,92 & 0,80 & 24 & 35 & 0,76 & 14,68 & 24,47 \\
\hline 20 & 201 & 0,99 & 0,92 & 0,76 & 0,66 & 18 & 35 & 0,76 & 9,03 & 20,34 \\
\hline 21 & 144 & 0,64 & 0,57 & 0,32 & 0,25 & 24 & 35 & 0,76 & 4,60 & 8,47 \\
\hline 22 & 156 & 0,71 & 0,64 & 0,39 & 0,32 & 18 & 25 & 0,76 & 4,35 & 7,45 \\
\hline 23 & 129 & 0,56 & 0,49 & 0,24 & 0,19 & 16 & 22 & 0,76 & 2,27 & 4,07 \\
\hline 24 & 211 & 1,05 & 0,98 & 0,87 & 0,75 & 18 & 35 & 0,76 & 10,33 & 23,05 \\
\hline 25 & 239 & 1,24 & 1,17 & 1,21 & 1,08 & 24 & 35 & 0,76 & 19,66 & 32,20 \\
\hline 26 & 311 & 1,77 & 1,70 & 2,46 & 2,27 & 25 & 35 & 0,76 & 43,12 & 65,44 \\
\hline 27 & 269 & 1,45 & 1,38 & 1,65 & 1,50 & 24 & 33 & 0,76 & 27,34 & 41,50 \\
\hline 28 & 80 & 0,32 & 0,25 & 0,08 & 0,05 & 18 & 28 & 0,76 & 0,65 & 1,66 \\
\hline 29 & 166 & 0,76 & 0,69 & 0,46 & 0,38 & 18 & 28 & 0,76 & 5,17 & 9,75 \\
\hline 30 & 82 & 0,32 & 0,25 & 0,08 & 0,05 & 18 & 26 & 0,76 & 0,70 & 1,64 \\
\hline
\end{tabular}

Fuente: elaboración propia.

\footnotetext{
${ }^{1}$ Ingeniero Forestal, Mg. Sc. Economía. Docente-Investigador de la Universidad Técnica Particular de Loja (UTPL). San Cayetano Alto - Loja, Ecuador. jabonilla2@utpl.edu.ec.

2 Doctor en Economía Aplicada. Profesor Principal, Facultad de Economía y Planificación, Universidad Nacional Agraria La Molina. Avenida La Molina S/N- La Molina, Apdo. 12-056- Lima 12, Perú. jalarcon@lamolina.edu.pe.
} 